\title{
Size and Shell Effects on the Photoacoustic and \\ Luminescence Properties of Dual Modal Rare-Earth Doped Nanoparticles for Infrared Photoacoustic Imaging
}

\author{
Yang Sheng ${ }^{1}$, Lun-De Liao ${ }^{2,5}$, Aishwarya Bandla ${ }^{2,3}$, Yu-Hang Liu ${ }^{2,4}$, Nitish Thakor ${ }^{2,3,4}$, \\ Mei Chee Tan ${ }^{1, *}$
}

1. Engineering Product Development, Singapore University of Technology and Design, Singapore, 8 Somapah Road, Singapore 487372

2. Singapore Institute for Neurotechnology (SINAPSE), National University of Singapore, 28 Medical Drive, \#05-COR, Singapore 117456

3. Department of Biomedical Engineering, National University of Singapore, 21 Lower Kent Ridge Rd, Singapore 119077

4. Department of Electrical and Computer Engineering, National University of Singapore, 21 Lower Kent Ridge Rd, Singapore 119077

5. Institute of Biomedical Engineering and Nanomedicine, National Health Research Institutes, 35 Keyan Rd., Zhunan Town, Miaoli County 35053, Taiwan, R.O.C.

*To whom correspondence should be addressed. Email: meichee.tan@sutd.edu.sg 

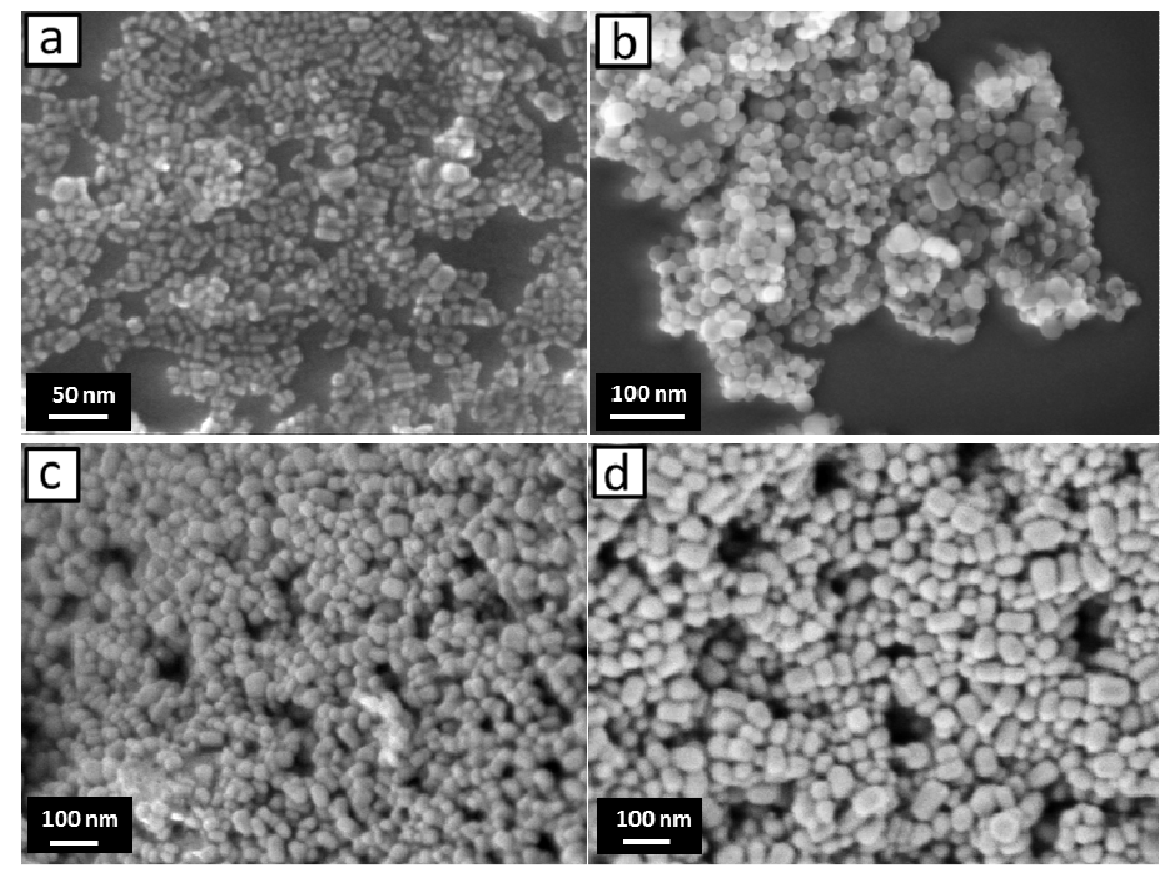

Figure S1 SEM images of core REDNPs of (a) RE(11) and (b) $R E(18)$ and corresponding core/shell REDNPs (c) RE(11)_NaYF 4 and (d) RE(18)_ NaYF 4 . All scale bars are $100 \mathrm{~nm}$. The corresponding seizes of as-prepared REDNPs are $11.4 \pm$ $3.1 \mathrm{~nm}$ and $18.4 \pm 3.2 \mathrm{~nm}$, while after coating the sizes increased to $29.1 \pm 5.6 \mathrm{~nm}$ and $36.8 \pm 6.5 \mathrm{~nm}$. 

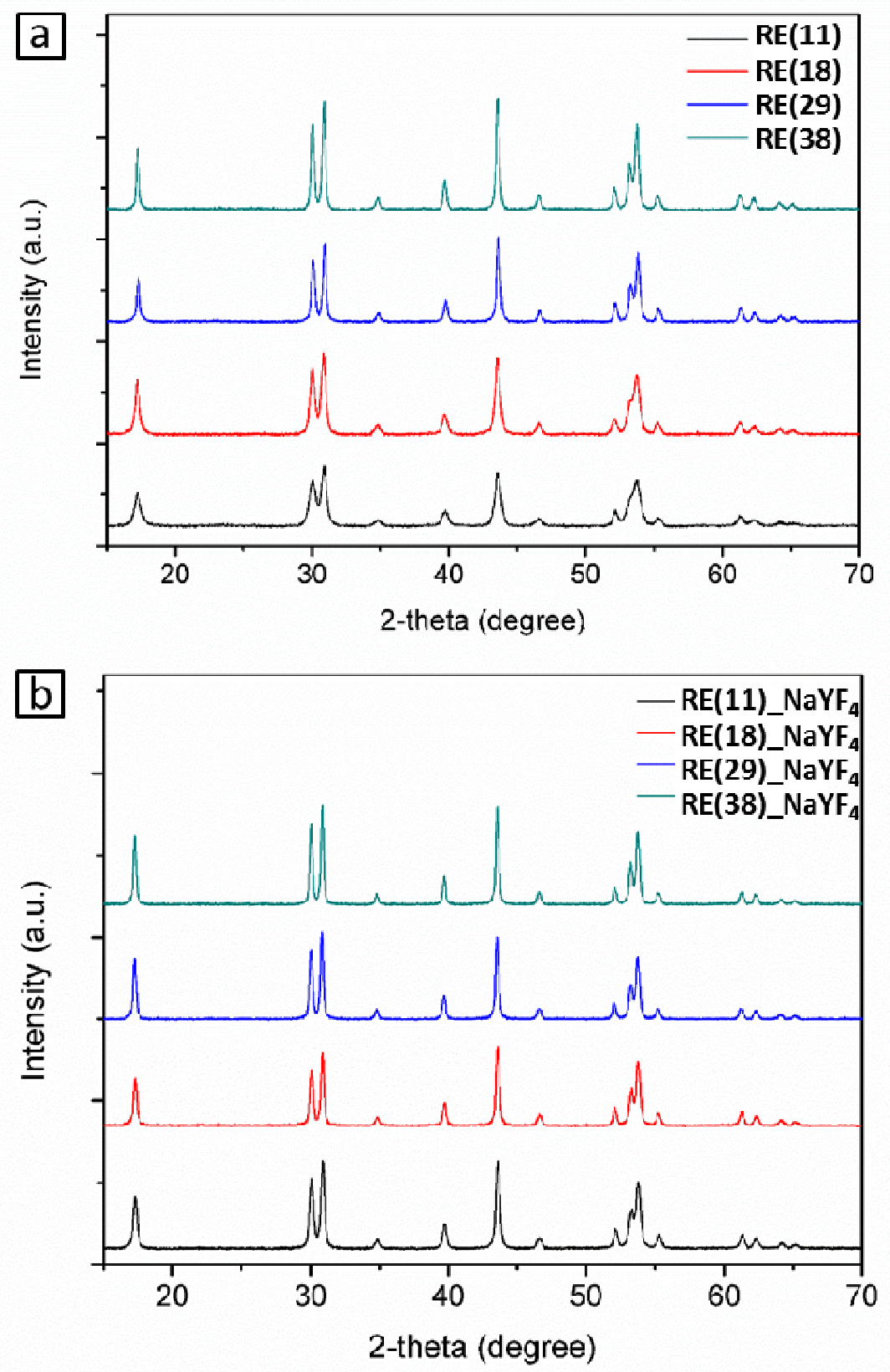

Figure S2 (a) XRD pattern of core REDNPs with different sizes. (b) XRD pattern of core/shell REDNPs with different sizes. 

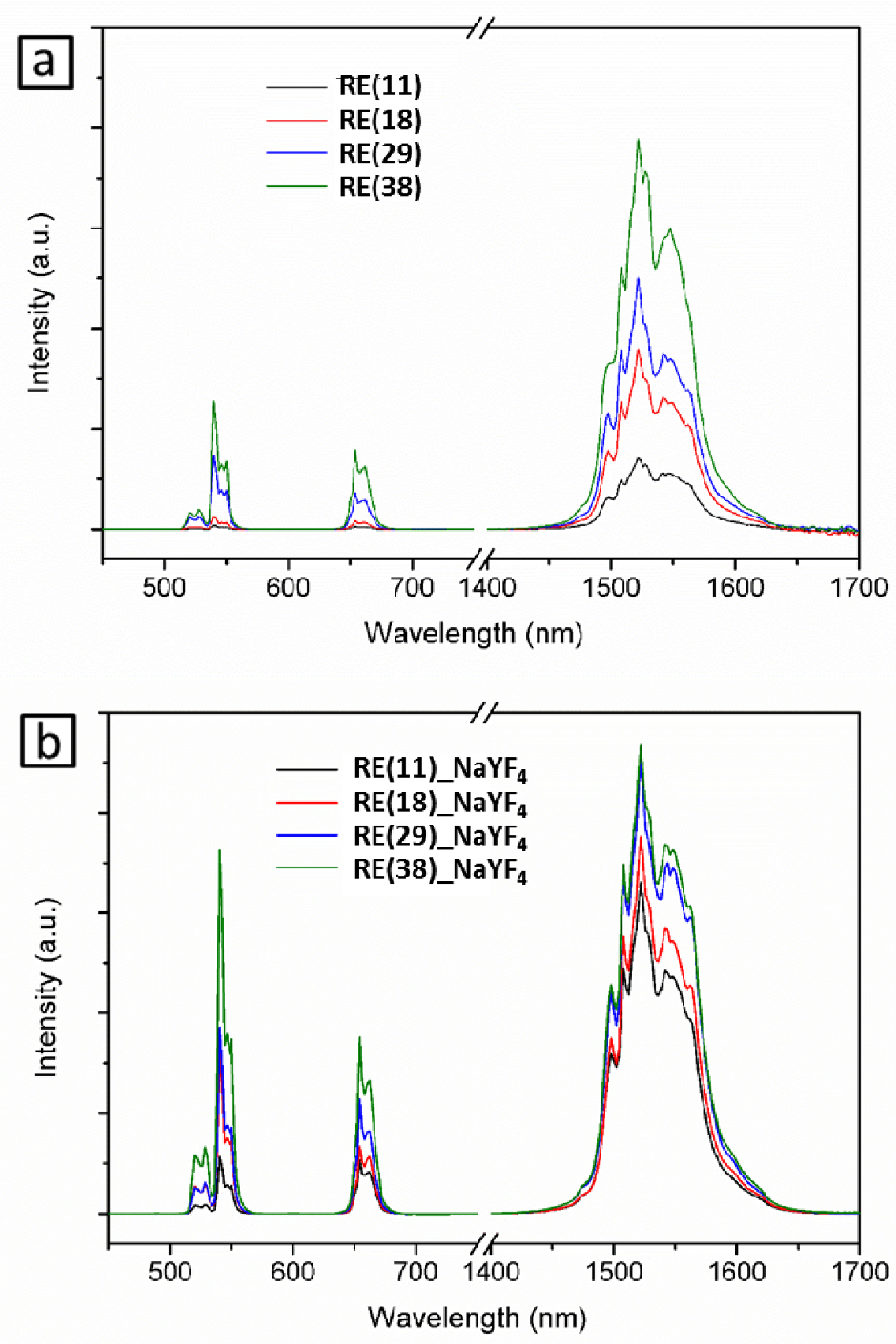

Figure S3 Steady state luminescence spectra of (a) core REDNPs and (b) core/shell REDNPs spanning visible and IR range. The line break in the $\mathrm{x}$-axis is due to the use of two different detectors required to measure the emissions in the visible (400 to 800 $\mathrm{nm}$ ) and infrared regions (1000 to $1700 \mathrm{~nm}$ ). The spectra were measured using continuous wave $(\mathrm{CW}) 975 \mathrm{~nm}$ laser at output power of $10 \mathrm{~mW}$. 

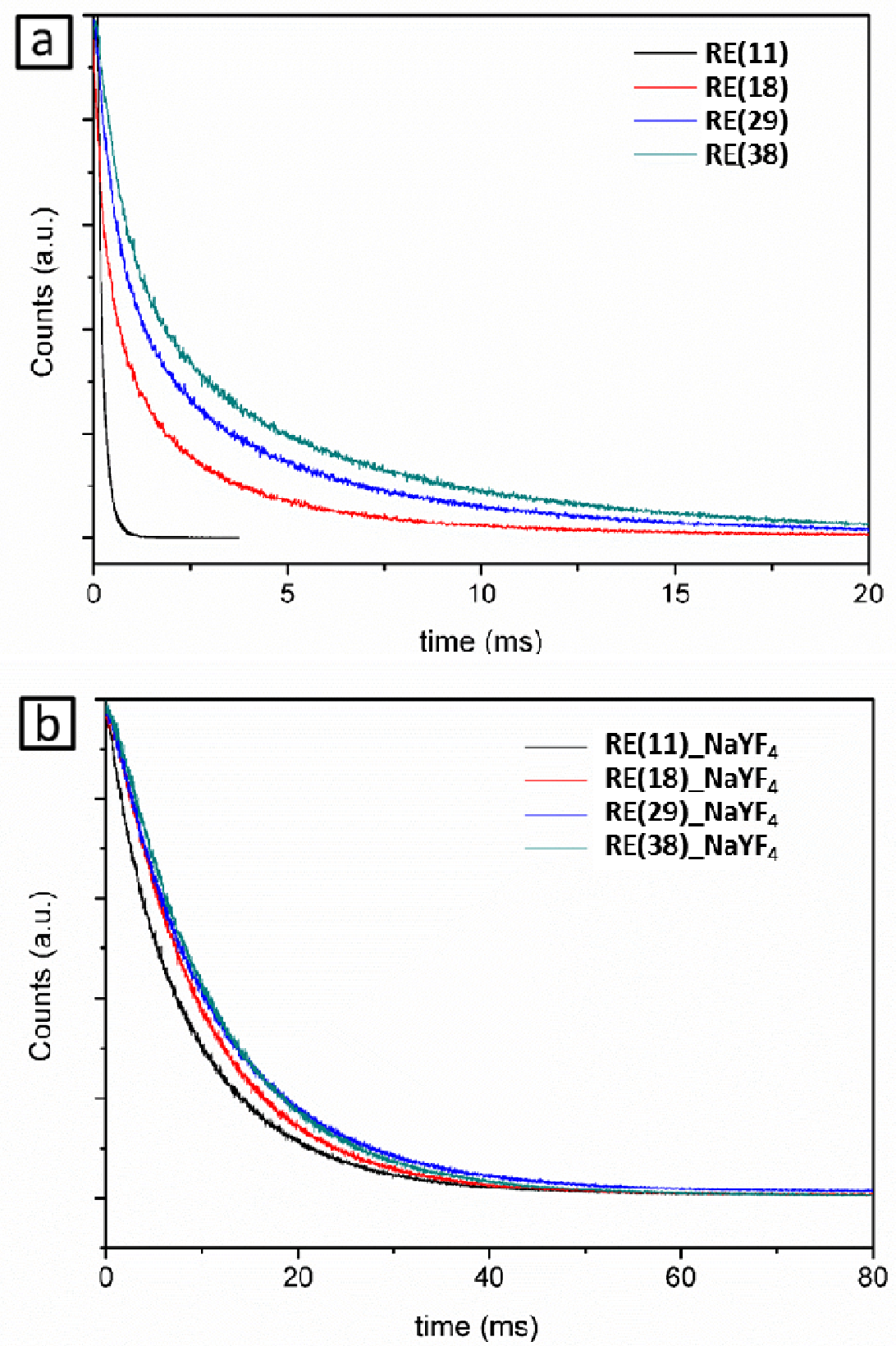

Figure S4 Time-resolved luminescence spectra of (a) core REDNPs and (b) core/shell REDNPs corresponding to the ${ }^{4} \mathrm{I}_{13 / 2}{ }^{4} \mathrm{I}_{15 / 2}$ transition of $\mathrm{Er}^{3+}$ at $1530 \mathrm{~nm}$. The CW 975 $\mathrm{nm}$ excitation source was modulated to measure the spectra using an electronic pulse modulator to obtain excitation pules at pulse duration of $20 \mu$ s with a repetition rate of $10 \mathrm{~Hz}$. 